\title{
Eldecalcitol improves chair-rising time in postmenopausal osteoporotic women treated with bisphosphonates
}

This article was published in the following Dove Press journal:

Therapeutics and Clinical Risk Management

16 January 2014

Number of times this article has been viewed

\author{
Jun Iwamoto' \\ Yoshihiro Sato ${ }^{2}$ \\ 'Institute for Integrated Sports \\ Medicine, Keio University School of \\ Medicine, Tokyo, Japan; ${ }^{2}$ Department \\ of Neurology, Mitate Hospital, \\ Fukuoka, Japan
}

Correspondence: Jun Iwamoto Institute for Integrated Sports Medicine, Keio University School of Medicine, 35 Shinanomachi, Shinjuku-ku, Tokyo 160-8582, Japan Tel +8I 33353 I2II (ext 62 I83)

Fax +8I 352699054

Email jiwamoto@a8.keio.jp
Abstract: An open-label randomized controlled trial was conducted to clarify the effect of eldecalcitol (ED) on body balance and muscle power in postmenopausal osteoporotic women treated with bisphosphonates. A total of 106 postmenopausal women with osteoporosis (mean age 70.8 years) were randomly divided into two groups ( $n=53$ in each group): a bisphosphonate group (control group) and a bisphosphonate plus ED group (ED group). Biochemical markers, unipedal standing time (body balance), and five-repetition chair-rising time (muscle power) were evaluated. The duration of the study was 6 months. Ninety-six women who completed the trial were included in the subsequent analyses. At baseline, the age, body mass index, bone mass indices, bone turnover markers, unipedal standing time, and chair-rising time did not differ significantly between the two groups. During the 6-month treatment period, bone turnover markers decreased significantly from the baseline values similarly in the two groups. Although no significant improvement in the unipedal standing time was seen in the ED group, compared with the control group, the chair-rising time decreased significantly in the ED group compared with the control group. The present study showed that ED improved the chair-rising time in terms of muscle power in postmenopausal osteoporotic women treated with bisphosphonates.

Keywords: osteoporosis, fall, vitamin D, muscle power, body balance

\section{Introduction}

Osteoporosis most commonly affects postmenopausal women, placing them at a significant risk for fractures. Osteoporotic fractures in women include vertebral fractures, hip fractures, wrist-forearm fractures, humeral fractures, rib fractures, pelvic fractures, clavicular, scapular, and sternum fractures, and tibial and fibular fractures. ${ }^{1}$ Because most non-vertebral osteoporotic fractures occur as a result of falls, risk factors for falls, including physical function, should be evaluated as part of the treatment of postmenopausal osteoporosis.

Clinically, the impairment of muscle strength and muscle power of the lower extremities, balance/postural control, and walking ability have been recognized as important risk factors for falls., ${ }^{2,3}$ However, muscle strength should be distinguished from muscle power: muscle strength is defined as the maximal force that a muscle can produce against a given resistance, while muscle power is defined as the product of force and speed. ${ }^{2,3}$ The former is related to bone strength, whereas the latter is related to falling. ${ }^{2-5}$ Thus, the evaluation of muscle power, rather than muscle strength, is likely to be important for the prediction of falls. Theoretically, flexibility, body balance, muscle power of the lower extremities, and walking ability should, at a minimum, be assessed in clinical practice. ${ }^{6}$ In particular, body balance can be evaluated by measuring 
unipedal standing time, while muscle power can be evaluated by measuring chair-rising time.,

Vitamin D is known to improve the volume of type II fibers in the muscle. ${ }^{8}$ An observational prospective study on a cohort of patients at an increased risk for falls showed that alfacalcidol ( $1 \alpha$-hydroxyvitamin $\mathrm{D}_{3}, 1 \mu \mathrm{g}$ daily) improved muscle power (chair-rising time) and balance (timed up and go and tandem standing time) and reduced the number of fallers (48.1\% reduction) and falls (51.3\% reduction). ${ }^{9}$ A meta-analysis study showed that alfacalcidol and calcitriol (1,25-dihydroxyvitamin $\left.\mathrm{D}_{3}\right)$ significantly reduced non-vertebral fractures (odds ratio: 0.51 ) and falls (odds ratio: 0.66 ) in the elderly. ${ }^{10}$ These reports suggest that the conventional active forms of vitamin $\mathrm{D}_{3}$ are useful for preventing falls in the elderly.

Recently, eldecalcitol [ED-71; $2 \beta$-(3-hydroxypropyloxy)1,25-dihydroxyvitamin $\mathrm{D}_{3}$ ] was generated as a new active orally administered vitamin $\mathrm{D}_{3}\left(1,25\right.$-dihydroxyvitamin $\left.\mathrm{D}_{3}\right)$ analog in Japan. A randomized, active comparator (alfacalcidol), double-blind study showed that ED (0.75 $\mu \mathrm{g}$ daily) was more effective than alfacalcidol ( $1 \mu \mathrm{g}$ daily) for suppressing bone turnover, increasing the lumbar spine and total hip bone mineral density (BMD), and preventing vertebral fractures in patients with osteoporosis. ${ }^{11}$ A subgroup analysis revealed that ED reduced the incidence of wrist fractures by $71 \%$ compared with alfacalcidol, ${ }^{11}$ suggesting that ED might have effectively prevented falls. However, the effect of ED on physical function and the incidence of falls remain to be established in patients with osteoporosis. Bisphosphonates (alendronate and risedronate) are standard medicines used for the treatment of postmenopausal osteoporosis. Thus, an open-label randomized controlled trial was conducted to clarify the effect of ED on body balance (unipedal standing time) and muscle power (chair-rising time) in postmenopausal osteoporotic women treated with bisphosphonates (alendronate and risedronate).

\section{Subjects and methods Study subjects}

Informed consent was obtained from all the participants. The protocol was approved by the Ethical Committee of Keiyu Orthopaedic Hospital (Gunma, Japan).

A total of 106 Japanese women (mean age 70.8 years, range 48-87 years) were recruited in three outpatient clinics in Japan (Keiyu Orthopaedic Hospital, Gunma; Hiyoshi Medical Clinic, Kanagawa; Ibata Orthopaedic Clinic, Chiba) during the 4-month period between April 1 and July 31, 2012. The inclusion criteria were spontaneous menopause, a fully ambulatory status, a diagnosis of postmenopausal osteoporosis according to the Japanese diagnostic criteria, ${ }^{12,13}$ an osteoporosis treatment-naïve status, and the feasibility of measuring the physical function parameters. The exclusion criteria for the physical function assessment were severe gait disturbance requiring some form of walking aid, current clinical fractures because of osteoporosis, sciatica because of lumbar spinal canal stenosis, rheumatoid arthritis, and severe cardiovascular disease. Because large asymmetrical leg extension power deficits observed in women recovering from hip fractures are reportedly associated with a slow stairclimbing speed, ${ }^{14}$ a history of hip fracture was selected as an exclusion criterion. The exclusion criteria for the diagnosis of postmenopausal osteoporosis included glucocorticoid use, gastrectomy, renal failure, or bone diseases including cancerinduced bone loss because of aromatase inhibitors, primary hyperparathyroidism, hyperthyroidism, Cushing's syndrome, multiple myeloma, Paget's disease of the bone, osteogenesis imperfecta, or type 2 diabetes. None of the subjects had been laborers or had been engaged in any regular or leisure-time sporting activities.

The participants were randomly divided into two groups: a bisphosphonate group ( $\mathrm{n}=53$, control group) and a bisphosphonate plus ED (0.75 $\mu \mathrm{g}$ daily) group ( $\mathrm{n}=53, \mathrm{ED}$ group). Alendronate (35 mg weekly) was used at Keiyu Orthopaedic Hospital, and risedronate (17.5 mg weekly) was used at Hiyoshi Medical Clinic and Ibata Orthopaedic Clinic. The doses in parentheses were approved by the Health, Labor, and Welfare Ministry in Japan. The study period was 6 months. Age, height, bodyweight, body mass index, history of non-vertebral fracture after menopause, bone mass, and physical function (unipedal standing time and chair-rising time) were assessed. Nonvertebral fractures included fractures at the distal radius, proximal humerus, rib, pelvis, clavicle, femur, and lower leg (a history of hip fracture was selected as an exclusion criterion). Thoracic and lumbar spine radiographs were obtained to assess prevalent vertebral fractures. Blood and urine samples were obtained for biochemical analysis, and the estimated glomerular filtration rate (eGFR) was also calculated.

During the 6-month treatment period, ten women dropped out from the study because of an acute phase reaction to bisphosphonates $(n=3)$, tooth extraction $(n=2)$, diarrhea $(n=1)$, the onset of rheumatoid arthritis $(n=1)$, a foot injury $(n=1)$, and surgery for a benign soft tissue tumor $(n=1)$ or venous varix $(n=1)$. Ninety-six women $(n=50$ in the ED group and $n=46$ in the control group) were included in the subsequent analyses. In the ED group, 26 women were treated with 
alendronate and 24 women were treated with risedronate. In the control group, 24 women were treated with alendronate and 22 women were treated with risedronate. The weekly dosing regimen was used for both bisphosphonates. The urinary cross-linked N-terminal telopeptides of type I collagen (NTX) levels were measured before and after 3 months of treatment, and the serum calcium, phosphorus, and alkaline phosphatase (ALP) levels were measured before and after 6 months of treatment. The unipedal standing time and chair-rising time were evaluated before and after 6 months of treatment. Changes in the biochemical markers, unipedal standing time, and chair-rising time during the 6-month treatment period were compared between the two groups.

\section{Japanese diagnostic criteria for postmenopausal osteoporosis}

All the patients had been diagnosed as having osteoporosis according to the Japanese diagnostic criteria. ${ }^{12,13}$ Namely, patients with a BMD $<70 \%$ of the young adult mean (YAM) or $70 \%-80 \%$ of the YAM along with a history of osteoporotic fractures were diagnosed as having osteoporosis.

At present, BMD measurement using dual-energy X-ray absorptiometry (DXA) is the most broadly established method of evaluating bone mass. Quantitative ultrasound (QUS) is a more recently developed noninvasive method of studying the bone density and structure in vivo. The QUS parameters can predict the risk of hip, wrist, and total non-vertebral fractures for up to 10 years. ${ }^{15}$ QUS might enable a better accounting of structural changes in the bone than the traditional DXA method. ${ }^{16}$ However, because the reliability of the speed of sound (SOS) of the calcaneus has not been fully established, the diagnosis of osteoporosis was made using both the SOS $(<70 \%$ of the YAM or $70 \%-80 \%$ of the YAM together with a history of osteoporotic fractures) and X-ray findings of the spine (ie, presence of osteopenia or possible osteopenia along with a history of osteoporotic fractures) when QUS was utilized.

\section{Assessment of morphometric vertebral fractures}

Plain lateral X-ray films of the thoracic and lumbar spine were obtained and examined for evidence of prevalent morphometric vertebral fractures. According to the Japanese criteria, a vertebral fracture was defined according to the vertebral height on lateral X-ray films. ${ }^{12,13}$ Briefly, the vertebral height was measured at the anterior (A), central (C), and posterior $(\mathrm{P})$ parts of the vertebral body, and the presence of a vertebral fracture was confirmed when: 1) a reduction in the vertebral height of more than $20 \%$ (A, C, and P) compared with the height of the adjacent vertebrae was observed, 2) the $\mathrm{C} / \mathrm{A}$ or $\mathrm{C} / \mathrm{P}$ was less than 0.8 , or 3 ) the $\mathrm{A} / \mathrm{P}$ was less than 0.75 . The assessment for vertebral fractures was performed at the T4-L4 level.

\section{Measurement of bone mass}

DXA, which is regarded as the gold standard, was available at the Keiyu Orthopaedic Hospital but not at either the Ibata Orthropaedic Clinic or the Hiyoshi Medical Clinic. The lumbar spine BMD was measured at the Keiyu Orthopaedic Hospital, the metacarpal BMD was measured at the Ibata Orthopaedic Clinic, and the calcaneus SOS was measured at the Hiyoshi Medical Clinic.

At the Keiyu Orthopaedic Hospital, the BMD of the lumbar spine (L1-L4) in the anteroposterior view was measured using DXA with a Hologic QDR 1500W apparatus (Hologic, Bedford, MA, USA). The coefficient of variation (CV) $(100 \times$ standard deviation [SD]/mean) of five measurements, with repositioning within 72 hours each time, was less than $1.2 \%$ among three persons.

At the Ibata Orthopaedic Clinic, the BMD of the metacarpus was measured using a computed X-ray densitometer (Teijin, Tokyo, Japan). An anteroposterior view radiograph of bilateral hands and an aluminum step wedge (20 steps, $1 \mathrm{~mm}$ per step) was obtained for each subject and was used as a standard. The BMD of the middle portion of the second metacarpus on the left hand was measured. The densitometer calculated the BMD based on the pattern expressed as graduations on the aluminum step wedge, and the BMD was expressed as the thickness of the aluminum equivalent $(\mathrm{mmAl})$ with a corresponding X-ray absorption. For these measurements, the $\mathrm{CV}$ for the intraobserver error (based on ten consecutive measurements by five persons), the daily change error (based on measurements performed on ten consecutive days), and the X-ray picturing error (based on ten exposures) were all within $1.3 \%-3.8 \%$.

At the Hiyoshi Medical Clinic, the SOS of the left calcaneus was measured using a QUS device (CM-200; Elk Corp, Osaka, Japan). The CV of five measurements, with repositioning within 24 hours each time, was $0.17 \%-0.43 \%$ among three healthy adults and $0.25 \%-0.35 \%$ among three osteoporotic patients.

\section{Blood and urinary biochemical tests and eGFR calculation}

Serum and urine samples were obtained from each patient, and the following biochemical analyses were performed. 
The serum calcium, phosphorus, ALP, aspartate aminotransferase, creatinine, hemoglobin, total protein, and albumin levels were measured using standard laboratory techniques. The urinary NTX level was measured using an ELISA (enzyme-linked immunosorbent assay). The eGFR was calculated using age and serum creatinine as follows: ${ }^{17}$ eGFR $\left(\mathrm{mL} / \mathrm{min} / 1.73 \mathrm{~m}^{2}\right)=194 \times$ serum creatinine $^{-1.094} \times$ age $^{-0.287} \times 0.739$.

\section{Assessment of physical function}

The unipedal standing time for the left leg was assessed as an index of body balance. Subjects stood on one leg for as long as possible, with their eyes open, without using any assistive device, and keeping both arms by their waist. This test was ended once 60 seconds had elapsed, ${ }^{18}$ when the stance of the foot shifted, when the lifted foot touched the floor, or when the hands detached from the waist. The patients were not allowed to press the lifted leg on to the standing leg.

The chair-rising time was assessed as an index of muscle power. The five-repetition chair-rising time was measured with the subject's arms crossed over her chest. The chair-rising time was the time required for the subjects to stand up from a chair (45 cm in height) five times as quickly as possible without using their arms, as measured using a stopwatch.

\section{Statistical analysis}

The per protocol set analysis was adopted. Data were expressed as the mean $\pm \mathrm{SD}$. An unpaired $t$-test was used to compare baseline anthropometry, bone mass, biochemical markers, eGFR, and physical function parameters, and the percentage changes in the biochemical markers and five-repetition chair-rising times between the two groups. The Fisher's exact test was used to compare the number of subjects with prevalent vertebral fractures and a history of non-vertebral fractures and the number of subjects divided by the unipedal standing time categories (0-29 seconds, 30-59 seconds, and 60 seconds) between the two groups. The Kaplan-Meier estimates and log-rank test were used to compare the unipedal standing time between the two groups. A one-way analysis of variance (ANOVA) with repeated measurements was used to examine longitudinal changes in the serum calcium, phosphorus, ALP, urinary NTX levels, and five-repetition chair-rising time within a group. A two-way ANOVA with repeated measurements was used to compare longitudinal changes in these parameters between the two groups. All the statistical analyses were performed using the Stat View J-5.0 program (SAS Institute, Cary, NC, USA) and Excel Statistics (Social Survey Research
Information Co, Ltd, Tokyo, Japan). The significance level was set at $P<0.01$ for all the comparisons.

\section{Results \\ Anthropometry, bone mass, prevalence of vertebral fractures, and history of non-vertebral fractures at baseline}

Table 1 shows that the mean age of the participants was 69.1 years in the control group and 72.3 years in the ED group. No significant differences in the baseline age, height, bodyweight, body mass index, bone mass parameters (lumbar spine BMD, metacarpal BMD, and calcaneus SOS), the number of subjects with prevalent vertebral fractures, or the number of subjects with a history of non-vertebral fractures were observed between the two groups. Eighteen women had a history of non-vertebral fracture at the distal radius $(n=9)$, proximal humerus $(n=2)$, rib $(n=4)$, or lower leg $(n=3)$, and all the fractures had completely healed, as assessed using plain radiographs.

None of the data regarding the anthropometry, bone mass, prevalence of vertebral fractures, and history of non-vertebral fractures at baseline differed significantly between the two

Table I Characteristics of study subjects

\begin{tabular}{|c|c|c|c|}
\hline & $\begin{array}{l}\text { Control group } \\
(n=46)\end{array}$ & $\begin{array}{l}\text { ED group } \\
(n=50)\end{array}$ & $P$-value \\
\hline Age, years & $69.1 \pm 9.9$ & $72.3 \pm 8.3$ & 0.0947 \\
\hline Height, m & $\mathrm{I} .5 \mathrm{I} \pm 0.06$ & $\mathrm{I} .5 \mathrm{I} \pm 0.05$ & 0.6292 \\
\hline Body weight, kg & $52.2 \pm 7.2$ & $51.1 \pm 7.1$ & 0.4897 \\
\hline Body mass index, $\mathrm{kg} / \mathrm{m}^{2}$ & $22.7 \pm 2.5$ & $22.5 \pm 3.3$ & 0.7398 \\
\hline $\begin{array}{l}\text { Lumbar spine BMD, } \\
\mathrm{g} / \mathrm{cm}^{2}\end{array}$ & $\begin{array}{l}0.682 \pm 0.090 \\
(n=24)\end{array}$ & $\begin{array}{l}0.624 \pm 0.114 \\
(n=26)\end{array}$ & 0.0537 \\
\hline $\begin{array}{l}\text { \% YAM in lumbar spine } \\
\text { BMD }\end{array}$ & $67.8 \pm 9.2$ & $61.6 \pm 11.4$ & 0.0396 \\
\hline Metacarpal BMD, mmAl & $\begin{array}{l}1.89 \pm 0.28 \\
(n=I I)\end{array}$ & $\begin{array}{l}|.8 I \pm 0.2| \\
(n=\mid 2)\end{array}$ & 0.4760 \\
\hline $\begin{array}{l}\text { \% YAM in metacarpal } \\
\text { BMD }\end{array}$ & $67.5 \pm 6.3$ & $63.5 \pm 7.2$ & 0.1704 \\
\hline Calcaneus SOS, $\mathrm{m} / \mathrm{sec}$ & $\begin{array}{l}I, 472 \pm 14 \\
(n=\mid I)\end{array}$ & $\begin{array}{l}I, 479 \pm 14 \\
(n=12)\end{array}$ & 0.2454 \\
\hline$\%$ YAM in calcaneus SOS & $67.1 \pm 6.5$ & $70.7 \pm 6.3$ & 0.2871 \\
\hline $\begin{array}{l}\text { Number }(\%) \text { of subjects } \\
\text { with prevalent vertebral } \\
\text { fractures }\end{array}$ & $16(34.8 \%)$ & 17 (34.0\%) & $>0.9999$ \\
\hline $\begin{array}{l}\text { Number }(\%) \text { of subjects } \\
\text { with history of non- } \\
\text { vertebral fractures }\end{array}$ & $6(13.0 \%)$ & $12(24.0 \%)$ & 0.1986 \\
\hline
\end{tabular}

Notes: Data are expressed as the mean \pm SD. An unpaired $t$-test and the Fisher's exact test were used to compare data between the two groups. Lumbar spine BMD was measured in 50 subjects, metacarpal BMD was measured in 23 subjects, and calcaneus SOS was measured in 23 subjects.

Abbreviations: BMD, bone mineral density; ED, eldecalcitol; NS, not significant; SD, standard deviation; SOS, speed of sound; YAM, young adult mean. 
groups, even though ten women who dropped out of the study were included in the analysis.

\section{Biochemical markers and eGFR at baseline}

Table 2 shows that the baseline hemoglobin, serum total protein, albumin, calcium, phosphorus, ALP, aspartate aminotransferase, creatinine, urinary NTX levels, and eGFR did not differ significantly between the two groups.

None of the data regarding the biochemical markers and eGFR at baseline differed significantly between the two groups, even though ten women who dropped out of the study were included in the analysis.

\section{Physical function parameters at baseline}

The mean baseline five-repetition chair-rising time in 96 women who were processed for the analyses was 10.1 seconds (range 4-26 seconds). The baseline fiverepetition chair-rising time did not differ significantly between the two groups (10.5 \pm 4.1 seconds in the ED group and $9.7 \pm 3.6$ seconds in the control group) and the baseline number $(\%)$ of subjects in each of the unipedal standing time categories (0-29 seconds, 30-50 seconds, and 60 seconds) did not differ significantly between the two groups (29 [58.0\%], 7 [14.0\%], and 12 [24.0\%], respectively, in the ED group and 21 [45.7\%], 8 [17.4\%], and 18 [39.1\%], respectively, in the control group). The Kaplan-Meier

Table 2 Biochemical markers and eGFR at baseline

\begin{tabular}{llccc}
\hline & $\begin{array}{l}\text { Normal } \\
\text { range }\end{array}$ & $\begin{array}{l}\text { Control } \\
\text { group } \\
(\mathbf{n}=\mathbf{4 6})\end{array}$ & $\begin{array}{l}\text { ED group } \\
(\mathbf{n}=\mathbf{5 0})\end{array}$ & P-value \\
\hline Serum & & & & \\
$\quad$ Hemoglobin, g/dL & $11.2-15.2$ & $12.9 \pm 1.0$ & $12.8 \pm 0.9$ & 0.5273 \\
Total protein, g/dL & $6.5-8.2$ & $7.3 \pm 0.6$ & $7.1 \pm 0.5$ & 0.1018 \\
Albumin, g/dL & $3.7-5.2$ & $4.2 \pm 0.3$ & $4.2 \pm 0.3$ & 0.3185 \\
Calcium, mg/dL & $8.4-10.2$ & $9.2 \pm 0.4$ & $9.2 \pm 0.4$ & 0.6876 \\
Phosphorus, mg/dL & $2.5-4.5$ & $3.3 \pm 0.5$ & $3.4 \pm 0.4$ & 0.1244 \\
ALP, IU/L & $100-340$ & $246 \pm 90$ & $261 \pm 94$ & 0.4154 \\
AST, IU/L & $11-35$ & $23.5 \pm 5.2$ & $23.0 \pm 6.0$ & 0.6925 \\
Cr, mg/dL & $0.47-0.79$ & $0.64 \pm 0.14$ & $0.63 \pm 0.12$ & 0.6679 \\
Urine & & & & \\
NTX (nmol & $9.3-54.3$ & $56.3 \pm 24.7$ & $56.1 \pm 23.9$ & 0.9710 \\
BCE $/ \mathrm{mmol} \mathrm{Cr})$ & & & & \\
eGFR, mL/min/ & $>60$ & $72.4 \pm 15.7$ & $72.9 \pm 17.3$ & 0.8918 \\
I.73 m & & & & \\
\hline
\end{tabular}

Notes: Data are expressed as the mean \pm SD. An unpaired $t$-test was used to compare data between the two groups.

Abbreviations: ALP, alkaline phosphatase; AST, aspartate aminotransferase; BCE, bone collagen equivalent; $\mathrm{Cr}$, creatinine; ED, eldecalcitol; eGFR, estimated glomerular filtration rate; NS, not significant; NTX, cross-linked N-terminal telopeptides of type I collagen; SD, standard deviation. estimates and the log-rank test showed no significant difference in the unipedal standing times between the two groups $\left(\chi^{2}=1.1348, P=0.2868\right)$.

None of the data regarding the physical function parameters at baseline differed significantly between the two groups, even though ten women who dropped out from the study were included in the analysis.

\section{Changes in biochemical markers related to calcium and bone metabolism}

Table 3 and Figure 1 show that the serum calcium levels did not change significantly from the baseline values in either group (one-way ANOVA). The serum phosphorus level did not change significantly from the baseline value in the ED group but increased significantly from the baseline value in the control group (one-way ANOVA). A significant difference in the changes in the serum phosphorus levels from the baseline values was observed between the two groups (two-way ANOVA). The serum ALP and urinary NTX levels decreased significantly from the baseline values in both groups (oneway ANOVA), but no significant differences were observed between the two groups (two-way ANOVA).

Table 4 shows that the mean rate of decrease in the urinary NTX level was $40.0 \%$ in the control group and $33.1 \%$ in the ED group. The respective mean rates of decrease in the serum ALP levels were $17.7 \%$ and $20.1 \%$. No significant differences in the percentage changes in serum ALP and urinary NTX levels were observed between the two groups. A significant difference in the percentage changes in serum phosphorus level, but not serum calcium level, was observed between the two groups.

Table 3 One-way and two-way ANOVA with repeated measurements ( $P$-values)

\begin{tabular}{|c|c|c|c|}
\hline & \multicolumn{2}{|c|}{ One-way ANOVA } & \multirow{2}{*}{$\frac{\text { Two-way ANOVA }}{\text { Control vs ED }}$} \\
\hline & Control group & ED group & \\
\hline \multicolumn{4}{|l|}{ Serum } \\
\hline Calcium & 0.5970 & 0.6092 & 0.8670 \\
\hline Phosphorus & 0.0015 & 0.3794 & 0.0035 \\
\hline ALP & $<0.0001$ & $<0.0001$ & 0.2328 \\
\hline \multicolumn{4}{|l|}{ Urine } \\
\hline NTX & $<0.0001$ & $<0.0001$ & 0.5366 \\
\hline $\begin{array}{l}\text { Five-repetition } \\
\text { chair-rising time }\end{array}$ & 0.0875 & $<0.0001$ & 0.0007 \\
\hline $\begin{array}{l}\text { Notes: A one-way } \\
\text { longitudinal changes } \\
\text { five-repetition chair } \\
\text { measurements was } \\
\text { between the two gr } \\
\text { Abbreviations: A } \\
\text { eldecalcitol; NS, no } \\
\text { collagen. }\end{array}$ & $\begin{array}{l}\text { In the serum calcium, } \\
\text {-rising time within a } \\
\text { used to compare } \\
\text { oups. } \\
\text { NOVA, analysis of } \\
\text { significant; NTX, cro }\end{array}$ & $\begin{array}{l}\text { ed measureme } \\
\text { shosphorus, AL } \\
\text { oup. A two-wa } \\
\text { ngitudinal chan } \\
\text { ariance; ALP, } \\
\text { s-linked N-tern }\end{array}$ & $\begin{array}{l}\text { nts was used to examine } \\
\text { P, urinary NTX levels, and } \\
\text { y ANOVA with repeated } \\
\text { ges in these parameters } \\
\text { lkaline phosphatase; ED, } \\
\text { inal telopeptides of type I }\end{array}$ \\
\hline
\end{tabular}




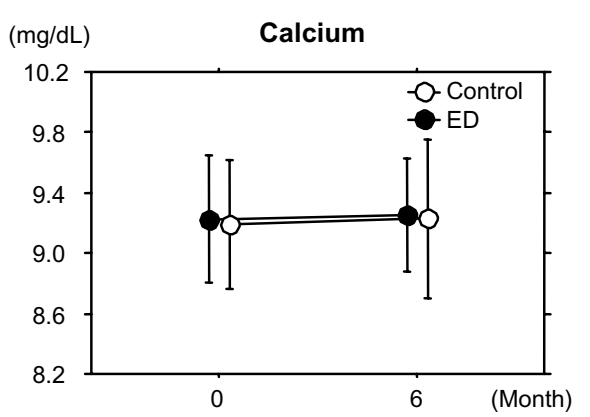

$$
\text { (IU/L) }
$$

ALP

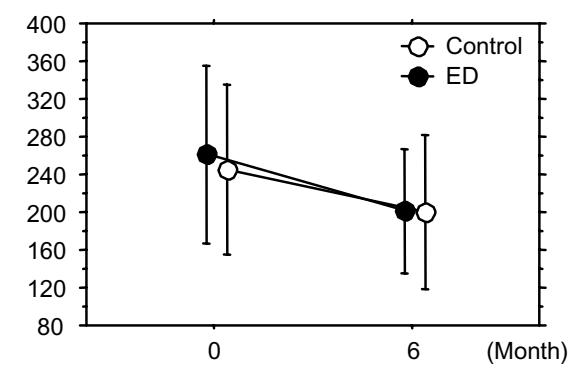

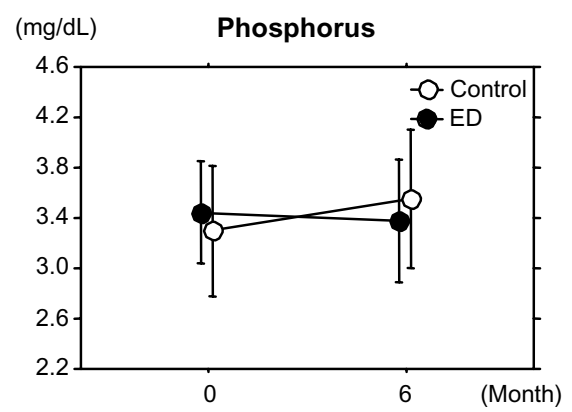

(nmol BCE/mmol Cr) Urinary NTX

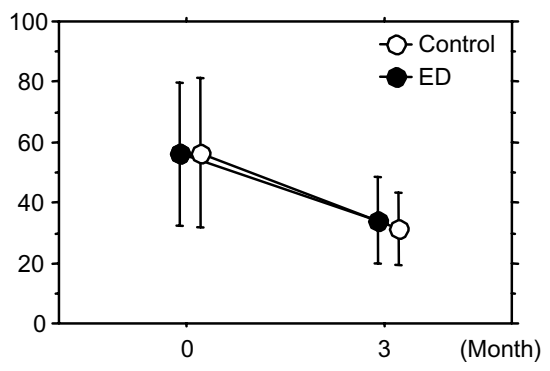

Figure I Changes in biochemical markers related to calcium and bone metabolism.

Notes: A one-way ANOVA with repeated measurements was used to examine longitudinal changes in the serum calcium, phosphorus, ALP, and urinary NTX levels within a group. A two-way ANOVA with repeated measurements was used to compare longitudinal changes in these parameters between the two groups.

Abbreviations: ALP, alkaline phosphatase; ANOVA, analysis of variance; BCE, bone collagen equivalent; Cr, creatinine; ED, eldecalcitol; NTX, cross-linked N-terminal telopeptides of type I collagen.

\section{Changes in physical function parameters}

Table 3 and Figure 2 show that the five-repetition chair-rising time did not change significantly in the control group (from 9.7 seconds to 9.2 seconds) but decreased significantly in the ED group (from 10.5 seconds to 8.6 seconds) (one-way ANOVA). A significant difference in the changes in the fiverepetition chair-rising time relative to the baseline value was seen between the two groups (two-way ANOVA).

Table 4 shows that the mean rate of decrease in the fiverepetition chair-rising time was $2.8 \%$ in the control group and $16.3 \%$ in the ED group. A significant difference in the

Table 4 Percentage changes in biochemical markers and fiverepetition chair-rising time

\begin{tabular}{lrrr}
\hline & Control group & ED group & P-value \\
\hline Serum & & & \\
$\quad$ Calcium & $0.56 \pm 5.63$ & $0.37 \pm 3.82$ & 0.8530 \\
$\quad$ Phosphorus & $8.3 \pm 15.4$ & $-1.17 \pm 14.2$ & 0.0024 \\
$\quad$ ALP & $-17.7 \pm 18.0$ & $-20.1 \pm 17.9$ & 0.5192 \\
Urine & & & \\
$\quad$ NTX & $-40.0 \pm 20.4$ & $-33.1 \pm 28.7$ & 0.1780 \\
Five-repetition & $-2.8 \pm 18.4$ & $-16.3 \pm 16.0$ & 0.0002 \\
chair-rising time & & & \\
\hline
\end{tabular}

Notes: Data are expressed as the mean $\pm \mathrm{SD}$. An unpaired $t$-test was used to compare data between the two groups.

Abbreviations: ALP, alkaline phosphatase; ED, eldecalcitol; NS, not significant; NTX, cross-linked $\mathrm{N}$-terminal telopeptides of type I collagen; SD, standard deviation. percentage changes in the five-repetition chair-rising time was seen between the two groups.

The number of subjects among the unipedal standing time categories (0-29 seconds, 30-50 seconds, and 60 seconds) after 6 months of treatment did not differ significantly between the two groups (28 [56.0\%], 6 [12.0\%], and 16 [32.0\%], respectively, in the ED group and 23 [50.0\%], 15 [32.6\%], and 8 [17.4\%], respectively, in the control group). The Kaplan-Meier estimates and log-rank test showed no significant difference in the unipedal standing time after 6 months of treatment between the two groups $\left(\chi^{2}=0.9994, P=0.3175\right)$.

\section{Adverse events}

As mentioned above, during the 6-month treatment period, ten women dropped out of the study. However, neither fallrelated fractures nor severe adverse events were observed in any of the participants.

\section{Discussion}

We conducted an open-label randomized controlled trial to clarify the effect of ED on physical function (muscle power index, chair-rising time; and body balance index, unipedal standing time) in postmenopausal osteoporotic women treated with bisphosphonates. Although no significant 


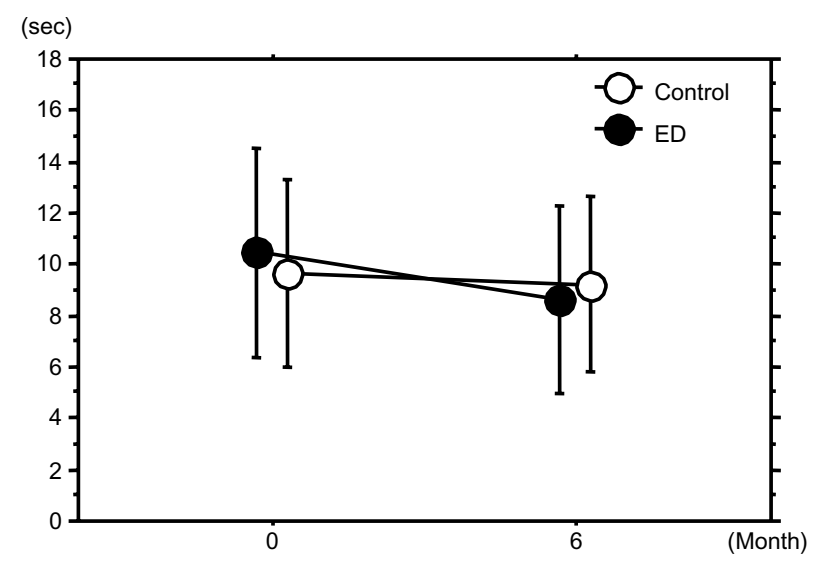

Figure 2 Changes in five-repetition chair-rising time.

Notes: A one-way ANOVA with repeated measurements was used to examine longitudinal changes in the five-repetition chair-rising time within a group. A twoway ANOVA with repeated measurements was used to compare longitudinal changes in this parameter between the two groups.

Abbreviations: ANOVA, analysis of variance; ED, eldecalcitol; sec, seconds.

improvement in the unipedal standing time was seen in the ED group, compared with the control group, the fiverepetition chair-rising time decreased significantly in the ED group (from 10.5 seconds to 8.6 seconds), compared with the control group (from 9.7 seconds to 9.2 seconds). These results suggested that ED improved the muscle power of postmenopausal osteoporotic women treated with bisphosphonates.

Previously, we reported that the five-repetition chairrising time was longer in postmenopausal osteoporotic women with a history of non-vertebral fracture than in those without such fractures. ${ }^{19} \mathrm{~A}$ randomized controlled trial showed that the chair-rising exercise (muscle power training) was more effective than the one-leg standing exercise (static balance training) for improving dynamic body balance, probably because the chair-rising exercise improved the function of the quadriceps and gluteus medius muscles and the joint movement of the lower extremities more effectively than a one-leg standing exercise in patients with locomotive disorders. ${ }^{20}$ Another randomized controlled trial also showed that power training improved balance, particularly using a low-load, high-velocity regimen, in older adults. ${ }^{21}$ The Osteo-cise: Strong Bones for Life study provided information on the efficacy of a targeted multi-modal community-based exercise program incorporating high-velocity resistance training together with osteoporosis education and a behavioral change program for improving multiple risk factors for falls and fractures. ${ }^{22}$ Thus, muscle power should be one of the key factors in strategies for preventing falls in the elderly.

An observational study demonstrated that 6 months of alfacalcidol treatment improved the parameters of the timed up and go, chair-rising test, and tandem standing test, leading to a decrease in the absolute numbers of fallers and falls among elderly adults, with a creatinine clearance of $\leq 65 \mathrm{~mL} / \mathrm{min}$. ${ }^{9}$ Another observational study also showed that 6 months of alfacalcidol treatment improved the chairrising time and the parameters of the timed up and go and tandem gait test in older adults. ${ }^{23}$ These reports suggest that not only the chair-rising time (muscle power) but also the timed up and go (dynamic body balance) are key parameters in elderly subjects with an increased risk of falls, and that lateral balance, as evaluated by a tandem maneuver, is correlated with falls and fractures. The effects of alfacalcidol on the chair-rising time (muscle power) and the timed up and go (dynamic body balance) may be attributable to the actions of vitamin D, including alfacalcidol, on type II fibers (fast twitch) in the muscle. ${ }^{24} \mathrm{ED}$ treatment improved muscle power (chair-rising time) in the present study, suggesting that ED acts on type II fibers (fast twitch) in muscle tissue. This result partly explains the efficacy of ED against wrist fractures ${ }^{11}$ in terms of physical function related to the risk of falls and subsequent non-vertebral fractures in patients with osteoporosis. ${ }^{19}$

However, the unipedal standing time was not significantly improved in the ED group, compared with the control group. The reproducibility of the unipedal standing time measurement in terms of the reliability of this parameter may be a greater issue than the reproducibility of the five-repetition chair-rising time. The average value of two or three measurements should have been evaluated. Sihvonen et a ${ }^{25}$ reported that community-dwelling older adults with a history of hip fracture had poor balance control, as evaluated using the semitandem standing test with the subjects' eyes closed. Schacht and Ringe ${ }^{23}$ reported that successful performance in the muscle tests (timed up and go, chair-rising test, and tandem gait test) was associated with a lower risk of falls and nonvertebral fractures in elderly patients with a low bone mass. These results suggest a relationship among body balance evaluated using a tandem maneuver, the risk of falls, and the risk of non-vertebral fractures in older adults. Thus, the tandem gait test and the tandem standing test should have been evaluated in the present study. Further studies are needed to clarify the effect of ED on body balance as evaluated using a tandem maneuver in postmenopausal women with osteoporosis.

During the 6-month treatment period, the urinary NTX and serum ALP levels decreased from baseline in both groups, but these decreases did not differ significantly between the two groups. A Phase III trial demonstrated that ED decreased the urinary NTX and serum bone-specific ALP 
levels from the baseline values in patients with osteoporosis. ${ }^{11}$ The rate of decrease in the urinary NTX level and the serum bone-specific ALP level after 6 months of treatment were reported to be approximately $27 \%-28 \% .{ }^{11}$ This trial clearly showed the anti-resorptive action of ED. Thus, the oversuppression of bone turnover was speculated to occur in patients treated with bisphosphonates plus ED. However, a subgroup analysis of a Phase III trial revealed that ED just normalized, but did not overly suppress, bone turnover, regardless of the baseline bone turnover markers. ${ }^{26}$ In the present study, ED treatment did not appear to suppress bone turnover further in patients treated with bisphosphonates.

The limitations of the present study should be discussed. First, the method used to measure bone mass for the diagnosis of osteoporosis differed among the three outpatient clinics. DXA, which is regarded as the gold standard, was available only at one outpatient clinic, and other methods were utilized at the other two outpatient clinics. Therefore, the inclusion and exclusion of patients eligible for participation in the present study might have been somewhat biased. Second, we did not evaluate vitamin D insufficiency/deficiency by measuring the serum 25-hydroxyvitamin D levels. In Japan, vitamin D supplementation is uncommon, and the measurement of serum 25-hydroxyvitamin D levels is not covered by health insurance. Because low serum levels of 25-hydroxyvitamin D are correlated with an increased risk of falls, and vitamin D supplementation reduces the incidence of falls in the elderly, $8,27,28$ evaluating the vitamin D status is important. Third, the present study was not powered to test the effect of ED on the incidence of falls, and the significance of the decrease in the fiverepetition chair-rising time (1.9 seconds, from 10.5 seconds to 8.6 seconds) in the ED group remains uncertain. Further studies are needed to resolve these limitations.

In conclusion, an open-label randomized controlled trial was conducted to clarify the effect of ED (a new active vitamin $\mathrm{D}_{3}$ analog) on body balance (unipedal standing time) and muscle power (chair-rising time) in postmenopausal osteoporotic women treated with bisphosphonates. Although the changes in the unipedal standing time did not differ significantly between the two groups, the five-repetition chair-rising time decreased significantly in the ED group, compared with the control group. These results suggested that ED improved muscle power in postmenopausal osteoporotic women treated with bisphosphonates.

\section{Acknowledgments}

We thank Dr Mitsuyoshi Uzawa (Keiyu Orthopaedic Hospital, Gunma, Japan), Dr Tetsuya Takada (Hiyoshi Medical Clinic,
Kanagawa, Japan), and Dr Iwao Ibata (Ibata Orthopaedic Clinic, Chiba, Japan) for their contributions to this trial.

\section{Disclosure}

The authors report no conflicts of interest in this work.

\section{References}

1. Giangregorio LM, Leslie WD; Manitoba Bone Density Program. Time since prior fracture is a risk modifier for 10-year osteoporotic fractures. J Bone Miner Res. 2010;25:1400-1405.

2. Runge M, Rehfeld G, Resnicek E. Balance training and exercise in geriatric patients. J Musculoskel Neuronal Interact. 2001;1:61-65.

3. Runge M, Hunter G. Determinants of musculoskeletal frailty and the risk of falls in old age. J Musculoskel Neuronal Interact. 2996;6: 167-173.

4. Frost HM. Defining osteopenias and osteoporosis: another view (with insights from a new paradigm). Bone. 1997;20:385-391.

5. Schiessl H, Frost HM, Jee WSS. Estrogen and bone-muscle strength and mass relationship. Bone. 1998;22:1-6.

6. Iwamoto J, Suzuki H, Tanaka K, et al. Preventative effect of exercise against falls in the elderly: a randomized controlled trial. Osteoporos Int. 2009;20:1233-1240.

7. Iwamoto J, Sato Y, Takeda T, et al. Whole body vibration exercise improves body balance and walking velocity in postmenopausal osteoporotic women treated with alendronate: Galileo and Alendronate Intervention Trail (GAIT). J Musculoskelet Neuronal Interact. 2012;12: 136-143.

8. Sato Y, Iwamoto J, Kanoko T, et al. Low-dose vitamin D prevents muscular atrophy and reduces falls and hip fractures in women after stroke: a randomized controlled trial. Cerebrovasc Dis. 2005;20:187-192.

9. Dukas L, Schacht E, Runge M, et al. Effect of a six-month therapy with alfacalcidol on muscle power and balance and the number of fallers and falls. Arzneimittelforschung. 2010;60:519-525.

10. O'Donnell S, Moher D, Thomas K, et al. Systematic review of the benefits and harms of calcitriol and alfacalcidol for fractures and falls. J Bone Miner Metab. 2008;26:531-542.

11. Matsumoto T, Ito $M$, Hayashi $Y$, et al. A new active vitamin $D_{3}$ analog, eldecalcitol, prevents the risk of osteoporotic fractures - a randomized, active comparator, double-blind study. Bone. 2011;49:605-612.

12. Orimo H, Sugioka Y, Fukunaga M, et al. Diagnostic criteria of primary osteoporosis. J Bone Miner Metab. 1998;16:139-150.

13. Orimo H, Hayashi Y, Fukunaga $M$, et al. Diagnostic criteria for primary osteoporosis: year 2000 revision. J Bone Miner Metab. 2001;19: 331-337.

14. Portegijs E, Sipilä S, Rantanen T, et al. Leg extension power deficit and mobility limitation in women recovering from hip fracture. $A m J$ Phys Med Rehabil. 2008;87:363-370.

15. Fujiwara S, Sone T, Yamazaki K, et al. Heel bone ultrasound predicts non-spine fracture in Japanese men and women. Osteoporos Int. 2005;16:2107-2112.

16. Wüster C, Heilmann P, Pereira-Lima J, et al. Quantitative ultrasonometry (QUS) for the evaluation of osteoporosis risk: reference data for various measurement sites, limitations and application possibilities. Exp Clin Endocrinol Diabetes. 1998;106:277-288.

17. Matsuo S, Imai E, Horio M, et al; Collaborators developing the Japanese equation for estimated GFR. Revised equations for estimated GFR from serum creatinine in Japan. Am J Kid Dis. 2009;53:982-992.

18. Yoshimura N, Oka H, Muraki S, et al. Reference values for hand grip strength, muscle mass, walking time, and one-leg standing time as indices for locomotive syndrome and associated disability: the second survey of the ROAD study. J Orthop Sci. 2011;16:768-777.

19. Iwamoto J, Sato Y, Matsumoto H. Chair rising time is longer in postmenopausal women with history of nonvertebral fracture. J Musculoskel Neuron Interact. 2013;13:265-272. 
20. Yamashita F, Iwamoto J, Osugi T, et al. Chair rising exercise is more effective than one-leg standing exercise in improving dynamic body balance: a randomized controlled trial. J Musculoskelet Neuronal Interact. 2012;12:74-79.

21. Orr R, de Vos NJ, Singh NA, et al. Power training improves balance in healthy older adults. J Gerontol A Biol Sci Med Sci. 2006;61: 78-85.

22. Gianoudis J, Bailey CA, Sanders KM, et al. Osteo-cise: Strong Bones for Life: protocol for a community-based randomised controlled trial of a multi-modal exercise and osteoporosis education program for older adults at risk of falls and fractures. BMC Musculoskelet Disord. 2012;13:78.

23. Schacht E, Ringe JD. Alfacalcidol improves muscle power, muscle function and balance in elderly patients with reduced bone mass. Rheumatol Int. 2012;32:207-215.
24. Sørensen OH, Lund B, Saltin B, et al. Myopathy in bone loss of ageing: improvement by treatment with 1 alpha-hydroxycholecalciferol and calcium. Clin Sci (Ond). 1979;56:157-161.

25. Sihvonen S, Kulmala J, Kallinen M, et al. Postural balance and self-reported balance confidence in older adults with a hip fracture history. Gerontology. 2009;55:630-636.

26. Shiraki M, Saito H, Matsumoto T. Eldecalcitol normalizes bone turnover markers regardless of their pre-treatment levels. Curr Med Res Opin. 2012;28:1547-1552.

27. Suzuki T, Kwon J, Kim H, et al. Low serum 25-hydroxyvitamin D levels associated with falls among Japanese community-dwelling elderly. J Bone Miner Res. 2008;23:1309-1317.

28. Bischoff-Ferrari HA, Dawson-Hughes B, Staehelin HB, et al. Fall prevention with supplemental and active forms of vitamin D: a metaanalysis of randomised controlled trials. BMJ. 2009;339:b3692.
Therapeutics and Clinical Risk Management

\section{Publish your work in this journal}

Therapeutics and Clinical Risk Management is an international, peerreviewed journal of clinical therapeutics and risk management, focusing on concise rapid reporting of clinical studies in all therapeutic areas, outcomes, safety, and programs for the effective, safe, and sustained use of medicines. This journal is indexed on PubMed Central, CAS,

\section{Dovepress}

EMBase, Scopus and the Elsevier Bibliographic databases. The manuscript management system is completely online and includes a very quick and fair peer-review system, which is all easy to use. Visit http://www.dovepress.com/testimonials.php to read real quotes from published authors.

Submit your manuscript here: http://www.dovepress.com/therapeutics-and-clinical-risk-management-journal 